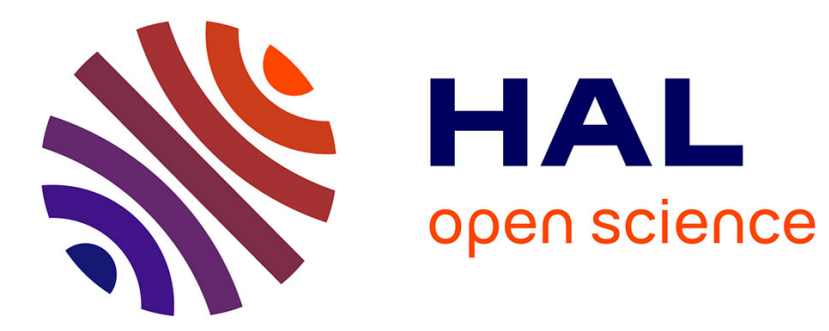

\title{
Geometric degree of non conservativeness
}

Jean Lerbet, Noël Challamel, François Nicot, Félix Darve

\section{To cite this version:}

Jean Lerbet, Noël Challamel, François Nicot, Félix Darve. Geometric degree of non conservativeness. 3rd International Conference on Geometric Science of Information (GSI 2017), Nov 2017, Paris, France. pp.355-358, 10.1007/978-3-319-68445-1_41 . hal-01658202

\section{HAL Id: hal-01658202 https://hal.science/hal-01658202}

Submitted on 11 Nov 2019

HAL is a multi-disciplinary open access archive for the deposit and dissemination of scientific research documents, whether they are published or not. The documents may come from teaching and research institutions in France or abroad, or from public or private research centers.
L'archive ouverte pluridisciplinaire HAL, est destinée au dépôt et à la diffusion de documents scientifiques de niveau recherche, publiés ou non, émanant des établissements d'enseignement et de recherche français ou étrangers, des laboratoires publics ou privés. 


\title{
Geometric Degree of Non Conservativeness
}

\author{
Jean Lerbet ${ }^{1(\bowtie)}$, Noël Challamel ${ }^{2}$, François Nicot $^{3}$, and Félix Darve ${ }^{4}$ \\ 1 IBISC Laboratory, Univ Evry, Paris-Saclay University, Évry, France \\ jlerbet@gmail.com \\ 2 LIMATB, South Brittain University, Lorient, France \\ 3 IRSTEA, Grenoble, France \\ 4 3SR, Grenoble Alpes University, Grenoble, France
}

\begin{abstract}
Symplectic structure is powerful especially when it is applied to Hamiltonian systems. We show here how this symplectic structure may define and evaluate an integer index that measures the defect for the system to be Hamiltonian. This defect is called the Geometric Degree of Non Conservativeness of the system. Darboux theorem on differential forms is the key result. Linear and non linear frameworks are investigated.
\end{abstract}

Keywords: Hamiltonian system · Symplectic geometry · Geometric Degree of Non Conservativeness · Kinematic constraints

\section{Position of the Problem}

Beyond the hamiltonian framework where external actions (like gravity) and internal actions (like in elasticity) may be described by a potential function, we are concerned here by mechanical systems whose actions are positional but without potential. For external actions, this is the case for example of the socalled follower forces ([1] for example). For internal actions, this is the case of the so-called hypoelasticity ([8] for example). One main characteristic of these questions is the loss of symmetry of the stiffness matrix $K(p)$ in the investigated equilibrium configuration and for the load parameter $p$.

For such systems, the stability issue presents some interesting paradoxical properties. For example, a divergence stable equilibrium configuration can become unstable as the system is subjected to appropriate additional kinematic constraints (see $[4,7]$ for example). This problem and the associate Kinematic Structural Stability concept have been deeply investigated for some years mainly in the linear framework ([3,4] for example). In the present work, we are concerned by the dual question: for such a non conservative system $\Sigma$, what is the minimal number of additional kinematic constraints that transform the non conservative system into a conservative one? This minimal number $d$ is called the geometric degree of nonconservativeness (GDNC). The second issue consists in finding the set of appropriate constraints. This issue will be tackled in the framework of discrete mechanics. More precisely, the set of configurations is a $n$-dimensional manifold $\mathbb{M}$ and the non hamiltonian actions are described by a section $\omega$ of 
the cotangent bundle $T^{*} \mathbb{M}$. This one form $\omega$ is supposed to be a non closed one form: $\mathbf{d} \omega \neq 0$ where $\mathbf{d}$ is the usual exterior derivative of differential forms. With the differential geometry concepts, the geometric meaning of the GDNC issue is: What is the highest dimension $n-d$ of embedded submanifolds $\mathbb{N}$ of $\mathbb{M}$ such that the "restriction" (in a well defined meaning) $\omega_{\mathbb{N}}$ to $\mathbb{N}$ is closed. We do not tackled in this work the very difficult global issues on $\mathbb{N}$ and, by Poincaré's theorem, the closed form $\omega_{\mathbb{N}}$ will be locally exact.

\section{Solution}

\subsection{Linear Framework}

In this subsection, we are concerned by the linearized version of the general problem. A configuration $m_{e} \in \mathbb{M}$ (we can think to $m_{e}$ as an equilibrium position) is fixed and a coordinate system $q=\left(q_{1}, \ldots, q_{n}\right)$ is given. We are looking for solution of the linear GDNC issue at $m_{e}$. There is here a real geometric issue to build the linearized counterpart of $\omega$ at $m_{e}$ because it should be obtained by derivative of $\omega$. But there is no connection on $\mathbb{M}$ to make the derivative of $\omega$. We will come back to this problem in the last part. However, as usual, in a coordinate system $q$, the linearized counterpart of $\omega$ is the so-called stiffness matrix $K=K\left(q_{e}\right)$ of the system at $m_{e}$ whose coordinate system is $q_{e}$.

In this framework, the issue is pulled back on the tangent space $T_{m_{e}} \mathbb{M}$ which will be identified with $\mathbb{R}^{n}$ thanks to the natural basis of $T_{m_{e}} \mathbb{M}$ associated with the coordinate system $q$ on $\mathbb{M}$. We indifferently note $E=\mathbb{R}^{n}$ and $E^{*}$ its dual space, the vector space of the linear forms on $E$. Thus, let $\phi$ the exterior 2 -form defined on $E=\mathbb{R}^{n}$ by:

$$
\phi(u, v)=u^{T} K_{a} v
$$

where $K_{a}$ is the skew-symmetric part of $K$. Usual linear algebra says that there is a basis $\mathcal{B}=\left(e_{1}, \ldots, e_{n}\right)$ of $\mathbb{R}^{n}$ and a number $r=2 s \leq n$ such that $\phi\left(e_{2 i-1}, e_{2 i}\right)=$ $-\phi\left(e_{2 i}, e_{2 i-1}\right)=1$ for $i \leq s$ and $\phi\left(e_{i}, e_{j}\right)=0$ for the other values of $i$ and $j$. In the dual basis $\left(e_{1}^{*}, \ldots, e_{n}^{*}\right)$ of $\left(e_{1}, \ldots, e_{n}\right)$, the form $\phi$ then reads:

$$
\phi=e_{1}^{*} \wedge e_{2}^{*}+\ldots+e_{2 s-1}^{*} \wedge e_{2 s}^{*}
$$

The solution of the linear GDNC issue at $m_{e}$ is then given by the following:

Proposition 1. $d=s$ is the GDNC of the mechanical system $\Sigma$ and a possible set $\mathcal{C}=\left\{C_{1}, \ldots, C_{s}\right\}$ of linear kinematic constraints making the constrained system $\Sigma_{\mathcal{C}}$ conservative is such that $C_{i}$ is any in $\left\langle e_{2 i-1}^{*}, e_{2 i}^{*}\right\rangle$ for $i=1, \ldots, s$.

In this framework, it is possible to find the set of all such possible constraints. Let then $F$ be the kernel of $\phi$. Then $\left(\mathbb{R}^{n} / F, \tilde{\phi}\right)$ is a $2 s$-dimensional symplectic vector space where $\tilde{\phi}$ is canonically defined by $\tilde{\phi}(\bar{u}, \bar{v})=\phi(x, y)$ with $x$ (resp, $y)$ any vector of the class $\bar{u}$ (resp. $\bar{v}$ ).

Proposition 2. The set of solutions of the GDNC is (isomorphic with) the set of Lagrangian subspaces of $\left(\mathbb{R}^{n} / F, \tilde{\phi}\right)$.

One can find in [6] a concrete construction of this set and in [5] the proof of these results. 


\subsection{Non Linear Framework}

The key of the solution in the nonlinear framework is related to Darboux theorem about the class of 1-form and 2-forms ([2] for example). We suppose now that the 2 -form $\mathbf{d} \omega$ is regular on $\mathbb{M}$ meaning that its class $r$ is constant on $\mathbb{M}$. Then here, since the form $\mathbf{d} \omega$ is itself a closed form $\left(\mathbf{d}^{2}=0\right)$, its class is also equal to its rank and is even: $r=2 s . s$ is the unique number such that $(\mathbf{d} \omega)^{s} \neq 0$ and $(\mathbf{d} \omega)^{s+1}=0$. We then deduce that $2 s \leq n$.

Darboux's theorem gives the local modeling of $\mathbf{d} \omega$ on an open set $U$ of $\mathbb{M}$ and reads:

$$
\mathbf{d} \omega=\sum_{k=1}^{s} d y^{k} \wedge d y^{k+s}
$$

where $y^{1}, \ldots, y^{2 s}$ are $2 s$ independent functions on $U$. We then deduce the following

Proposition 3. Suppose that the class of $\mathbf{d} \omega$ is constant at $m \in \mathbb{M}$ (namely maximal). The (non linear) GDNC of $\Sigma$ (in a neighborhood of $m \in \mathbb{M}$ is then the half $s$ of the class $2 s$ of $\mathbf{d} \omega$. The local definition of a submanifold $\mathbb{N}$ solution of the problem is given by the family $f^{1}=0, \ldots, f^{s}=0$ of equations on $\mathbb{M}$ where $f^{i}$ is any linear combination (in the vector space on $\mathbb{R}$ and not in the modulus on the ring on the functions on $\mathbb{R}$ ) of the above $y^{i}$ and $y^{i+s}$ for all $i=1, \ldots, s$.

\section{Open Issues}

Two open issues are related to this GDNC issue. The first one concerns the derivative of sections in $T^{*} \mathbb{M}$. The dual issue is the KISS issue that involves, in a linearized version at $m_{e}$, the symmetric part $K_{s}\left(q_{e}\right)$ of the stiffness matrix $K\left(q_{e}\right)$. It is worth noting that the skew-symmetric aspect $K_{a}\left(q_{e}\right)$ may be extended to the nonlinear framework through the exterior derivative $\mathbf{d} \omega$ whereas no similar extension is possible for the symmetric part without specify a connection on $\mathbb{M}$. This issue is today partially solved and will be the subject of a forthcoming paper.

The second one concerns the extension to continuum mechanics and infinite dimension spaces. Regarding the dual KISS issue, it is has been performed and will be soon published in an already accepted paper. Regarding the GDNC issue, it remains an interesting challenge because the tools, involved for the finite dimensional solution, are not naturally extendable to the case of infinite dimensional (Hilbert) vector spaces.

\section{References}

1. Bolotin, V.V.: Nonconservative Problems of the Theory of Elastic Stability. Pergamon Press, London (1963)

2. Godbillon, C.: Géométrie différentielle et mécanique analytique. Herman, Paris (1969) 
3. Lerbet, J., Challamel, N., Nicot, F., Darve, F.: Variational formulation of divergence stability for constrained systems. Appl. Math. Model. doi:10.1016/j.apm.2015.02. 052

4. Lerbet, J., Challamel, N., Nicot, F., Darve, F.: Kinematical structural stability. Discr. Contin. Dyn. Syst. Ser. S (DCDS-S) 9(2), 529-536 (2016). American Institute of Mathematical Sciences (AIMS)

5. Lerbet, J., Challamel, N., Nicot, F., Darve, F.: Geometric degree of nonconservativity: set of solutions for the linear case and extension to the differentiable non linear case. Appl. Math. Modell. (2016). doi:10.1016/j.apm.2016.01.030

6. Souriau, J.M.: Construction explicite de l'indice de Maslov. Applications. In: Janner, A., Janssen, T., Boon, M. (eds.) Group Theoretical Methods in Physics. Lecture Notes in Physics, vol. 50. Springer, Heidelberg (1976). doi:10.1007/3-540-07789-8_13

7. Thompson, J.M.T.: Paradoxical mechanics under fluid flow. Nature 296(5853), 135$137(1982)$

8. Truesdell, C.: Hypoelasticity. J. Rationa. Mech. Anal. 4(83-133), 1019-1020 (1955) 